\title{
À la recherche des chronotopes du roman urbain. Une cartographie des Mystères de Bruxelles (1845-1846)
}

In search of urban novel chronotypes. A cartography of Mystères de Bruxelles (1845-1846)

Los cronotopos en la novela urbana. La cartografía de los Misterios de Bruselas (1845-1846)

Paul Aron, Laurence Brogniez, Tatiana Debroux, Jean-Michel Decroly et Christophe Loir

\section{(Q) OpenEdition}

\section{Journals}

Édition électronique

URL : http://journals.openedition.org/mappemonde/3592

DOI : 10.4000/mappemonde.3592

ISSN : 1769-7298

Éditeur

UMR ESPACE

Référence électronique

Paul Aron, Laurence Brogniez, Tatiana Debroux, Jean-Michel Decroly et Christophe Loir, « À la recherche des chronotopes du roman urbain. Une cartographie des Mystères de Bruxelles (1845-1846) », Mappemonde [En ligne], 121 | 2017, mis en ligne le 01 juillet 2017, consulté le 29 avril 2020. URL : http://journals.openedition.org/mappemonde/3592 ; DOI : https://doi.org/10.4000/ mappemonde.3592

Ce document a été généré automatiquement le 29 avril 2020. 


\section{À la recherche des chronotopes du roman urbain. Une cartographie des Mystères de Bruxelles (1845-1846)}

In search of urban novel chronotypes. A cartography of Mystères de Bruxelles (1845-1846)

Los cronotopos en la novela urbana. La cartografía de los Misterios de Bruselas (1845-1846)

Paul Aron, Laurence Brogniez, Tatiana Debroux, Jean-Michel Decroly et Christophe Loir

\section{NOTE DE L'AUTEUR}

Ce travail a été réalisé dans le cadre de l'Action de Recherche Concertée MICM-arc développée au sein de l'Université libre de Bruxelles et consacrée à l'analyse pluridisciplinaire des relations entre la culture, la mobilité et le territoire à travers le cas bruxellois $\left(18^{\mathrm{e}}-21^{\mathrm{e}}\right.$ siècles $)$. Voir http://micmarc.ulb.ac.be

\section{Introduction}

1 Dans le domaine littéraire, l'espace prend plusieurs significations. Il est d'abord le lieu même de déploiement du texte comme, en poésie, lorsque la disposition des vers sur la page participe de la « mise en scène » voulue par l'auteur. Dans le théâtre, par la nature même du genre, la parole se déploie toujours dans un espace visible, qui participe au sens même du propos. À un second niveau, l'espace est référence, investie par la subjectivité. Dans le roman, il devient souvent une sorte de protagoniste de l'action, soit par les lieux qui sont décrits ou suggérés, soit par ceux auxquels l'auteur fait référence en tant qu'art langagier, se développant dans le temps de son énoncé, le texte 
littéraire construit également un espace au second degré, dans la saisie totalisante qu'autorise la lecture rétrospective: le lecteur peut ainsi rapprocher des éléments séparés par le fil du récit et les organiser en «tableaux » mentaux ${ }^{1}$.

2 Le concept de chronotope, défini par le sémioticien russe Mikhail Bakhtine et affiné par plusieurs chercheurs après lui, a donné une réelle opérativité aux analyses de l'espace romanesque (Bakhtine, 1978) en les liant à la temporalité, et donc aux catégories fondamentales de l'entendement humain définies par Kant. Le chronotope désigne la fusion d'indices spatiaux et temporels en un tout intelligible et concret. Il peut se condenser dans une image (le seuil, la route, le salon), ou même devenir le propre d'une structure romanesque comme c'est le cas dans ce que Mikhail Bakhtine lui-même appelle le « roman grec » ou le roman de chevalerie ${ }^{2}$. Au final, le concept de chronotope constitue une invitation à porter attention aux "espaces-temps" structurant la matière même de la fiction, parce qu'ils permettent aux personnages de se rencontrer ou de faire avancer le récit.

3 Par ailleurs, depuis une dizaine d'années, les études de géographie littéraire prétendent incarner un véritable renouveau, notamment par les possibilités de représentation concrète qu'elles offrent à la matière du roman ${ }^{3}$. Il importe toutefois de vérifier l'apport spécifique de la cartographie littéraire, tout en examinant les difficultés auxquelles elle se heurte.

4 Nous proposons ici une « étude de cas » qui prend pour objet le roman des Mystères de Bruxelles (1845-1846) de Suau de Varennes. Rédigé par un Français émigré à Bruxelles, il illustre parfaitement la double ambition du roman urbain, à savoir un repérage matériel des lieux et une analyse de leur dimension symbolique (Aron, 2015). Notre ambition est de faire émerger, à différents niveaux de généralité, les chronotopes du roman de Suau. Pour ce faire, nous mobilisons une démarche cartographique, qui vise à représenter, à l'aide d'un système d'information géographique ${ }^{4}$, les lieux mentionnés dans Les Mystères sur des cartes contemporaines à leur rédaction.

Deux raisons au moins conduisent à mobiliser ici la cartographie thématique ${ }^{5}$. Tout d'abord, la mise en cartes des lieux mentionnés dans l'œuvre étudiée permet d'acquérir une vision synthétique et synoptique de l'espace dans lequel évoluent les personnages. En efFet, la carte rassemble sur une seule figure, de taille réduite (quelques dizaines de $\mathrm{cm}^{2}$ ), l'ensemble ou un sous-ensemble des lieux inventoriés. Elle offre donc la possibilité de révéler des structures qui ne sont pas directement visibles à la seule lecture du texte puisque dans ce dernier, les lieux apparaissent en ordre dispersé et leur localisation n'est connue que de ceux qui ont une bonne représentation mentale de l'espace urbain évoqué. Les spécificités de la perception visuelle facilitent ce processus de lecture globale, puisque l'œil perçoit d'abord un ensemble avant de chercher le détail (Zanin, 2006). Ensuite, dès lors que les mentions géoréférençables sont dénombrées, la cartographie permet de hiérarchiser les lieux du roman selon leur fréquence d'apparition et donc de dresser une géographie différentielle de l'espace romanesque, qui identifie des centres, des marges et des périphéries de l'action.

6 Nous expliquerons d'abord comment nous avons mis le roman "en cartes" et illustrerons l'apport de cette démarche par des cartes consacrées aux espaces narrativisés, à la mobilité et aux trajectoires des personnages. Cette étude conduit à redéfinir l'usage du chronotope à plusieurs niveaux. Le genre romanesque, en luimême, selon Mikhail Bakhtine, est un chronotope que l'on peut opposer à celui dont 
font usage d'autres genres, comme l'épopée ou la tragédie. À un second niveau, celui de l'espèce, le roman urbain qui apparaît au début du XIX siècle constitue une forme de saisie spécifique de la ville. La sous-espèce du roman des «Mystères de... » affine encore cette perception en insistant sur les tensions qui structurent la ville et sa périphérie et en donnant un véritable «mode d'emploi» de cette réalité contemporaine aux auteurs qui la décrivent ${ }^{6}$. Enfin une série de chronotopes particuliers révèlent la ville comme ensemble structuré : les quartiers; les micro-lieux pertinents (comme un magasin de tabac) et les différents modes de déplacement des acteurs sociaux. En conclusion, nous situerons notre démarche en regard des différentes manières dont s'opère le "tournant cartographique » dans le domaine littéraire et historique; nous montrerons que si le roman urbain s'était donné pour mission de rendre la ville lisible, l'analyse cartographique rend sa démarche visible.

\section{La « mise en cartes » du roman}

7 Quels sont les éléments du texte qu'il convient de retenir? En la matière, notre hypothèse repose sur un geste d'exhaustivité. Nous nous fondons sur le concept d' espace localisé pertinent : un lieu situé de manière compréhensible par le lecteur. Ces lieux sont tantôt référentiels, donc géolocalisables, et peuvent donc être reportés sur une carte d'époque, tantôt autoréférentiels, et donc relatifs à d'autres, voire totalement internes à la fiction (le logement d'un personnage) à condition qu'ils soient situés («à proximité de... »). Nous posons donc d'emblée quatre exigences :

- L'analyse sera donc totalisante: le recensement des lieux intègre les lieux fictifs autant que les lieux réels.

- Nous considérons qu'il faut recenser les lieux réels dans la base de données tout en réservant une mention spéciale à leurs connotations et à leur fonction dans la dynamique du texte. Dans notre approche dynamique, l'item de chapitrage et la distinction entre lieux principaux et secondaires permettent de respecter le rythme du roman.

- Il importe de tenir compte du contexte associé à chaque lieu : les lieux ne sont pas seulement des noms, mais portent souvent des enjeux symboliques ou économiques liés à l'histoire de la ville. Problématiser les données recueillies permet de leur donner du sens. Il est donc nécessaire d'adopter une approche contextuelle.

- Nous voulons produire des données qui seront réutilisables dans d'autres travaux portant sur la même ville, la même époque ou des œuvres littéraires comparables. Il importe donc que les données puissent être aisément récupérées pour une approche comparative.

Pour répondre à ces quatre exigences, nous constituons une base de données à partir d'un tableau Excel reprenant pour chaque mention à un lieu :

- sa localisation (au mieux l'adresse exacte, autrement le nom de la rue, du quartier ou de la commune);

- son importance dans le récit : principale (où l'action se déroule) ou secondaire (lieu cité) ; son ordre d'apparition dans l'ouvrage (partie, chapitre) ;

- les personnages évoqués lors de son apparition. 
Tableau 1. Aperçu partiel du tableau Excel du début du roman

\begin{tabular}{|c|c|c|c|c|c|c|c|c|c|c|c|c|}
\hline \multicolumn{3}{|c|}{ Identification dans récit } & \multicolumn{3}{|c|}{ Localisation } & \multicolumn{3}{|c|}{ Importance du lieu } & \multicolumn{4}{|c|}{ Personnages } \\
\hline Partie & Chapitre & Identifiant & Lieu & Localisation & Type__lieu & Importance & Nb_princ & Nb_sec & Louise de W & Lucien & Trinette & ... \\
\hline 1 & 1 & 10101 & $\begin{array}{l}\text { cité } \\
\text { Cluysenaar }\end{array}$ & $\begin{array}{l}\text { rue Royale, } \\
101-103\end{array}$ & Point & Principal & 4 & 2 & 1 & 1 & & \\
\hline 1 & 1 & 10102 & $\begin{array}{c}\text { Galeries du Roi } \\
\text { et de la Reine } \\
\text { (projet) }\end{array}$ & & Quartier & Secondaire & & 1 & & & & \\
\hline 1 & 2 & 10201 & $\begin{array}{l}\text { quartier } \\
\text { Léopodd }\end{array}$ & & Quartier & Secondaire & & 1 & & & & \\
\hline 1 & 2 & 10202 & $\begin{array}{l}\text { boulevard } \\
\text { d'Anvers }\end{array}$ & & Point & Secondaire & & 1 & & & & \\
\hline 1 & 2 & 10203 & $\begin{array}{l}\text { cité } \\
\text { Cluysenaar }\end{array}$ & & Point & Principal & & & 1 & 1 & & \\
\hline 1 & 2 & 10204 & Cercle du Bac & $\begin{array}{l}\text { rue de } \\
\text { l'Evêque }\end{array}$ & Pant & Secondaire & 3 & 2 & & & & \\
\hline 1 & 2 & 10205 & $\begin{array}{c}\text { Salon de } \\
\text { Mme d'Egmont }\end{array}$ & $\begin{array}{l}\text { prés de la } \\
\text { rue des Sols }\end{array}$ & Point & Secondaire & & 1 & & & & \\
\hline 1 & 2 & 10206 & $\begin{array}{c}\text { Hotel } \\
\text { Wladimont }\end{array}$ & $\begin{array}{l}\text { En face du } \\
\text { Jarch boganique } \\
\text { (bout } \\
\text { rue Royale) }\end{array}$ & Point & Secondaire & & & 1 & 1 & & \\
\hline 1 & 3 & 10301 & $\begin{array}{l}\text { Cour aux } \\
\text { seigles }\end{array}$ & $\begin{array}{l}\text { rue Rempart } \\
\text { du Nord }\end{array}$ & Quartier & Principal & 1 & & & & 1 & \\
\hline 1 & 3 & 10302 & $\begin{array}{c}\text { boulevard du } \\
\text { Jardin botanique }\end{array}$ & & Point & Secondaire & & 1 & & & & \\
\hline 1 & 3 & 10303 & $\begin{array}{l}\text { Place de } \\
\text { la Nonnaie }\end{array}$ & & Point & Secondaire & & 3 & & & & \\
\hline 1 & 3 & 10304 & Amigo & & Point & Secondaire & & 1 & & & & \\
\hline
\end{tabular}

9 Tous les lieux identifiés dans le roman et présents dans cette base de données ont été cartographiés ${ }^{7}$ et représentés sur des fonds de cartes géolocalisés contemporains au récit, de manière à faire apparaître l'espace des Mystères de Bruxelles.

\section{Un roman urbain}

10 À l'instar de leur prestigieux modèle français, Les Mystères de Bruxelles sont d'abord un roman urbain. Mais que faut-il entendre exactement par « roman urbain »? Une étude récente sur le sujet précise que tout en ayant pour cadre une grande ville, il « doit également s'ancrer dans l'époque contemporaine [à l'auteur et au lecteur au moment de la parution], s'intéresser au quotidien ordinaire de ses personnages et porter les marques intrinsèques de l'actualité » (Horvath C., 2008). Ces caractéristiques en font un sous-genre inscrit dans une double histoire : celle de la littérature qui voit apparaître les romans urbains dans le premier tiers du XIX ${ }^{e}$ siècle, et celle des villes, qui connaissent à ce moment d'importants travaux liés à la croissance démographique, l'hygiène et l'urbanisme.

\section{Un récit centré sur la ville}

11 Les chiffres tirés de la base de données permettent de se faire une idée plus précise du phénomène. Même si quelques scènes ont lieu hors de l'espace bruxellois (la plupart d'entre elles en périphérie), l'action de nos Mystères se concentre efrectivement dans la ville. Un inventaire chiffré confirme aisément cette intuition de lecture: 149 références spatiales sont localisées à Bruxelles, 37 en périphérie proche, 6 dans une périphérie plus éloignée, mais accessible aisément à cheval ou en calèche (Alsemberg et Cortenberg), et seulement 3 près de Gand (Wetteren), soit à plus de 30 kilomètres. 
12 La mise en cartes permet de préciser le genre. Toutefois on voudrait ici souligner qu'elle fait l'objet de choix graphiques autant que conceptuels qui demandent à être pensés par les chercheurs. Voyons en efFet les deux cartes suivantes :

Figure 1. Localisation simple - un point sur chaque lieu

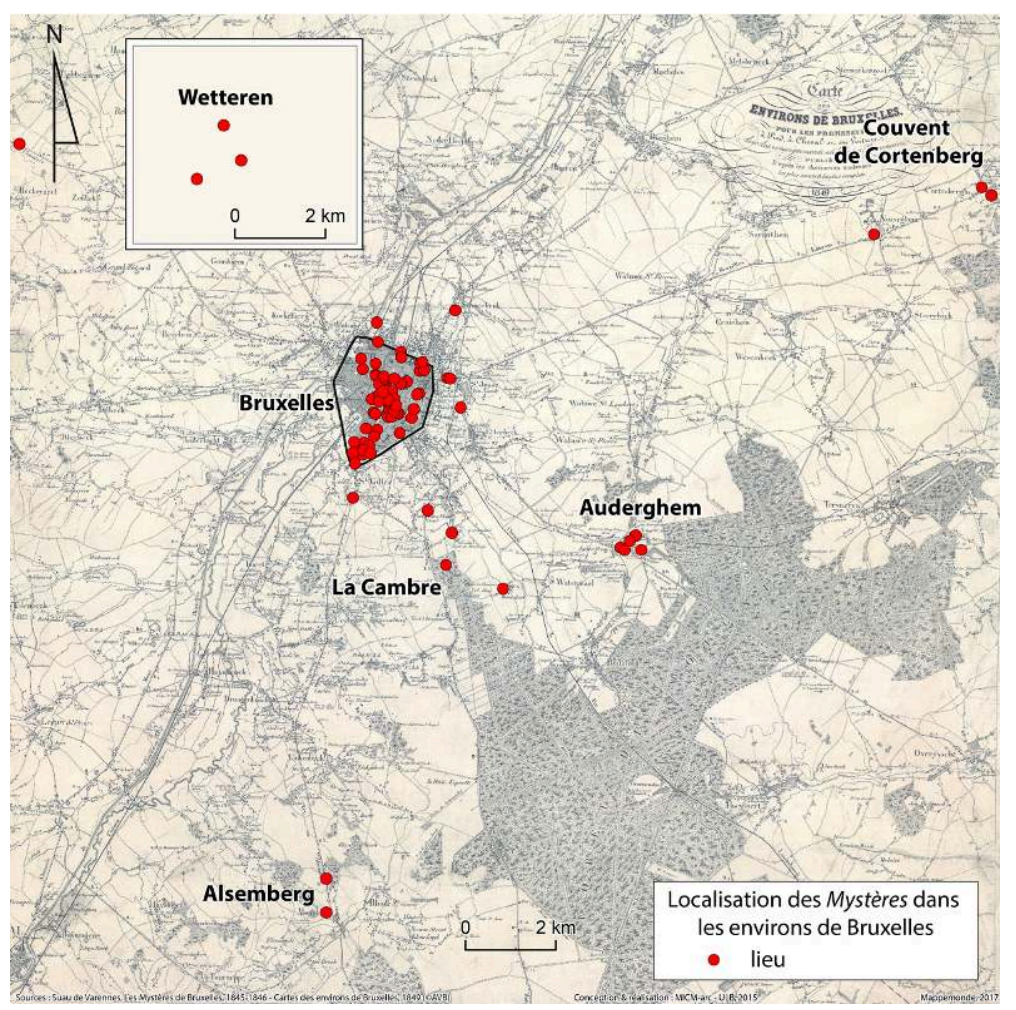


Figure 2. Importance des lieux - nombre d'apparitions et statut dans l'intrigue

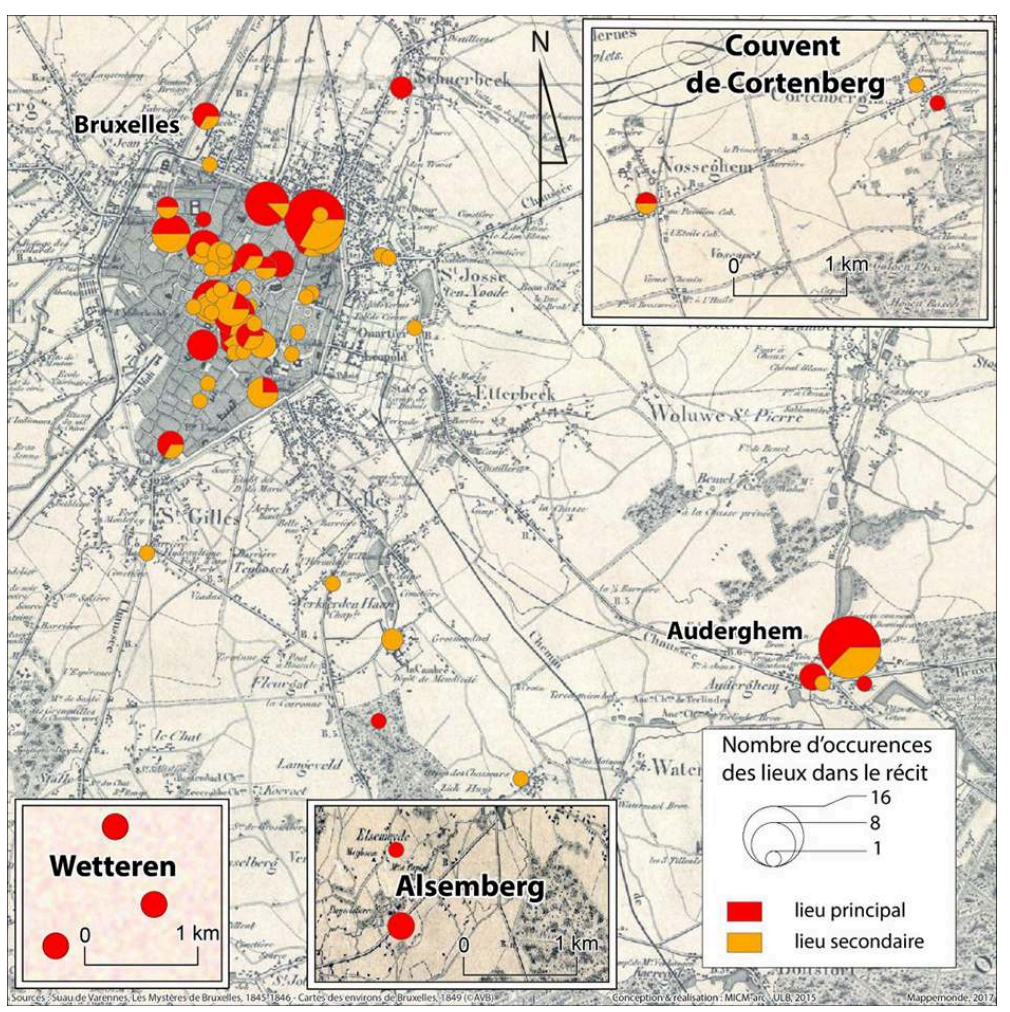

13 La comparaison indique sans ambigüité que le simple pointage des lieux cités est d'un moindre rendement que le repérage des lieux relativement au nombre d'occurrences et à leur importance dans l'intrigue. Si les deux illustrations précédentes montrent que l'espace bruxellois n'est pas exploité dans sa totalité, la seconde offre l'avantage de faire apparaître plus clairement les zones privilégiées par le matériau romanesque. Au sein du «Pentagone», appellation courante pour désigner le tracé de la seconde enceinte bruxelloise (XIVe siècle), les Mystères de Bruxelles font notamment référence à deux quartiers pauvres, l'un situé au sud, appelé les Marolles, l'autre dans le coin nord-est, baptisé Notre-Dame-aux-Neiges. Le quartier des Marolles se détache nettement sur la figure 1, car il fait l'objet de nombreuses références dans les lieux secondaires cités, en accord avec l'emphase d'un narrateur qui se présente comme un explorateur social. En revanche, il se marque moins sur la figure 2, car il est rarement le lieu même de l'action parce que peu de personnages sont liés aux rues de ce quartier dans la base de données. Une forte dimension fantasmatique n'équivaut donc pas à une importance fonctionnelle. Le quartier Notre-Dame-aux-Neiges, pour sa part, figure discrètement sur la carte du pointage des lieux, mais ressort comme un point focal de l'espace romanesque sur la figure 2. En efFet, comme nous le verrons dans la suite de l'analyse, Suau de Varennes confère une attention soutenue à ce quartier, qui incarne les mutations urbaines à l'œuvre au XIX ${ }^{\mathrm{e}}$ siècle à Bruxelles.

Les deux cartes indiquent clairement que l'action des Mystères de Bruxelles se concentre dans les quartiers du bas de ville qui relient le centre à la gare du Nord, puis dans l'espace central urbain qui remonte vers les «beaux quartiers » du haut de la ville et la porte de Namur (à l'est de la ville - voir la figure 3 pour la localisation précise). Les principaux espaces périphériques exploités dans le livre, vers Auderghem et le bois de 
la Cambre, confirment amplement cet axe. Le roman urbain n'est pas nécessairement un roman de toute la ville; il y opère des choix significatifs.

\section{Une plongée dans la ville banale}

15 Si le roman urbain se focalise sur la ville, il aborde cette dernière selon un angle singulier, puisqu'il vise à montrer le quotidien ordinaire de ses personnages. En ce sens, il devient un instrument de connaissance du réel.

16 La comparaison de la répartition spatiale des lieux des Mystères de Bruxelles et de celle des principaux monuments que recommande de visiter un guide touristique contemporain ${ }^{8}$ est éclairante à cet égard (figure 3). Elle montre l'opposition entre deux modèles de savoirs sur la ville. On voit bien que Suau a tenu à déplacer les centres d'intérêt classiques. Il excentre son propos jusqu'au nord du Canal, région ignorée des touristes, et la « visite » qu'il propose des quartiers dangereux se situe aux antipodes des axes fréquentés.

Figure 3. Carte des lieux de l'action

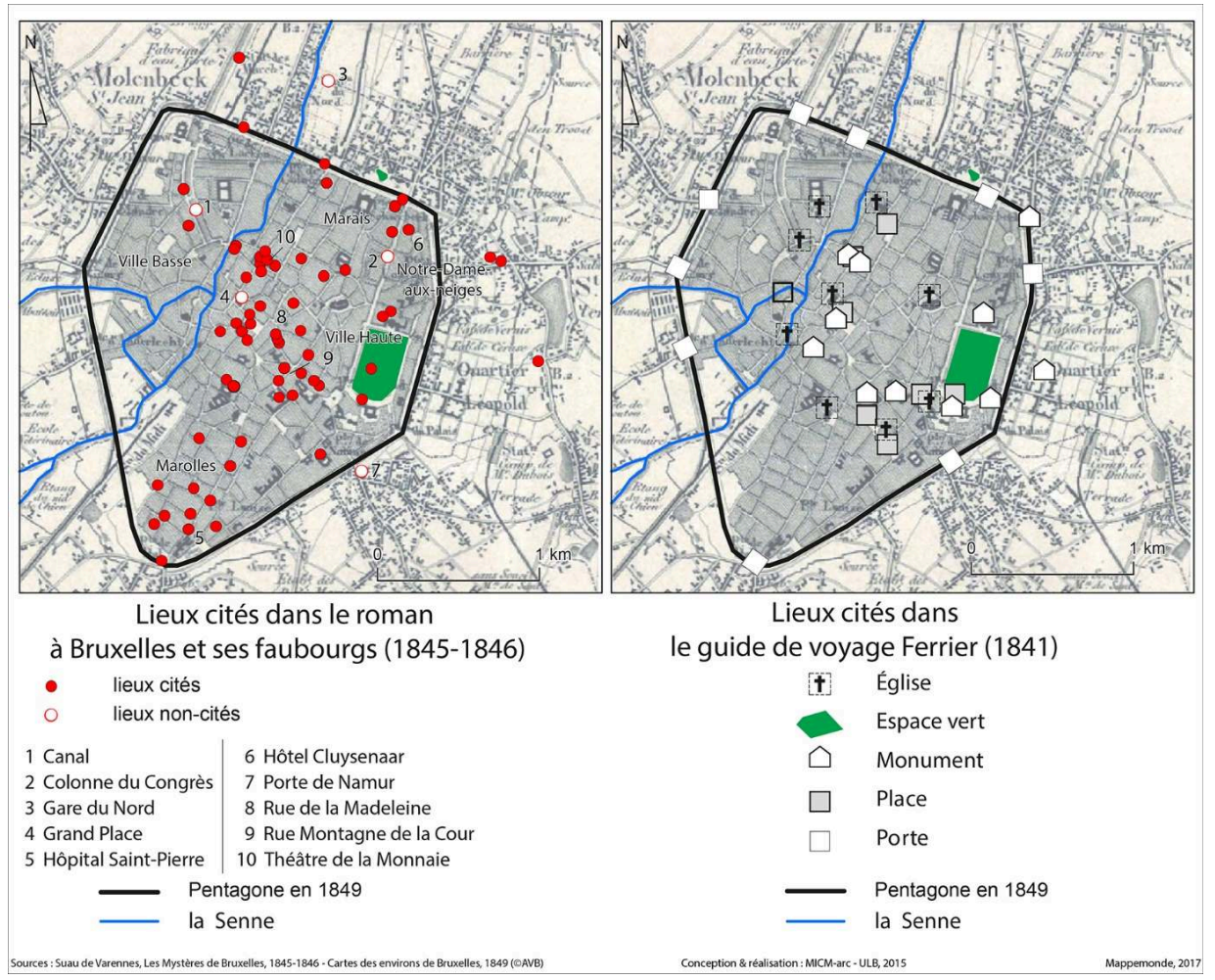

17 La comparaison des deux cartes permet de comprendre le ton du roman, qui se présente comme une sorte de guide inversé, une exploration des zones inconnues du tissu urbain. Le passage consacré au quartier Notre-Dame-aux-Neiges est le plus significatif (I, p. 59-60). Le lexique utilisé fait de la ville elle-même le sujet de la visite guidée : on « débouche » dans une rue, on en «aperçoit » une autre où l'on «jette un regard »; la « curiosité » nous meut, et, comme des naturalistes découvrant une espèce inconnue, nous pourrons " examiner en toute sûreté des bouges et des cloaques ».

Français expatrié en Belgique (parce qu'il est poursuivi par la justice de son pays), Suau a certainement consulté des guides sur la région où il s'est réfugié. Mais son savoir 
pratique est aussi lié à des contacts personnels. Il a certainement rencontré d'autres émigrés et des Belges, qui lui ont fait visiter Bruxelles. À la lecture des Mystères, on sent bien (et le narrateur le confirme d'ailleurs parfois) qu'il connait de première main l'hôpital Saint-Pierre, l'abbaye de la Cambre devenue hospice pour jeunes délinquants (au sud de la ville, voir la figure 1), les quartiers «dangereux », la Grand-Place, les cercles de jeu et les promenades des environs de la ville.

\section{Le chronotope des Mystères}

Le chronotope des Mystères urbains tel qu'Eugène Sue en fixe le modèle en 1842-1843 comporte plusieurs caractéristiques qui en font une sous-espèce particulière de roman. Fondé sur une exploration en profondeur de l'espace parisien, il met en scène la ville moderne en insistant sur son originalité historique. Il la définit comme un espace complexe, formé par la difFérenciation entre l'urbain et le périurbain, ainsi que par la différenciation sociale de ceux qui y habitent. Le roman s'attache dès lors à cerner précisément les personnages et leurs lieux d'habitation, qui sont traités pour euxmêmes, mais aussi en tant qu'éléments d'un système où leurs traits spécifiques fonctionnement dans un jeu d'oppositions et de ressemblances pertinentes. Le chronotope des Mystères insiste sur les «qualifications différentielles» des personnages ${ }^{9}$. Nous soulignerons en particulier la manière dont les Mystères de Bruxelles montrent la proximité spatiale de groupes sociaux difFérenciés tout en les distinguant par leurs lieux de résidence et par leur mobilité dans l'espace urbain.

\section{Bruxelles, un petit Paris}

20 Espace de rencontres et d'explorations, Bruxelles est présentée comme un analogue crédible à Paris, la ville par excellence qui définit à l'époque ce genre de littérature. Le lien est explicite dès le premier chapitre. Suau met en scène l'hôtel Cluysenaar, une maison, ou plutôt un ensemble de logements, qui fonctionne à la fois comme une synecdoque particularisante de la ville tout entière (et donc comme un chronotope thématique) et comme un terme de la comparaison entre Bruxelles et Paris. C'est pourquoi également la première partie des Mystères multiplie les ancrages spatiaux avec une insistance que l'on ne retrouvera plus par la suite : sur les 12 chapitres qu'elle comporte, 6 sont désignés par des noms de lieux.

La base de données permet de suivre le fil du texte pour dessiner précisément la dynamique spatiale du récit, qui privilégie d'abord le centre urbain pour s'autoriser ensuite de nombreux allers-retours avec la périphérie, dilatant progressivement l'espace du roman (excepté la partie 4 qui voit l'action se replier sur le centre-ville). 
Figure 4. Carte schématique des lieux de l'action romanesque

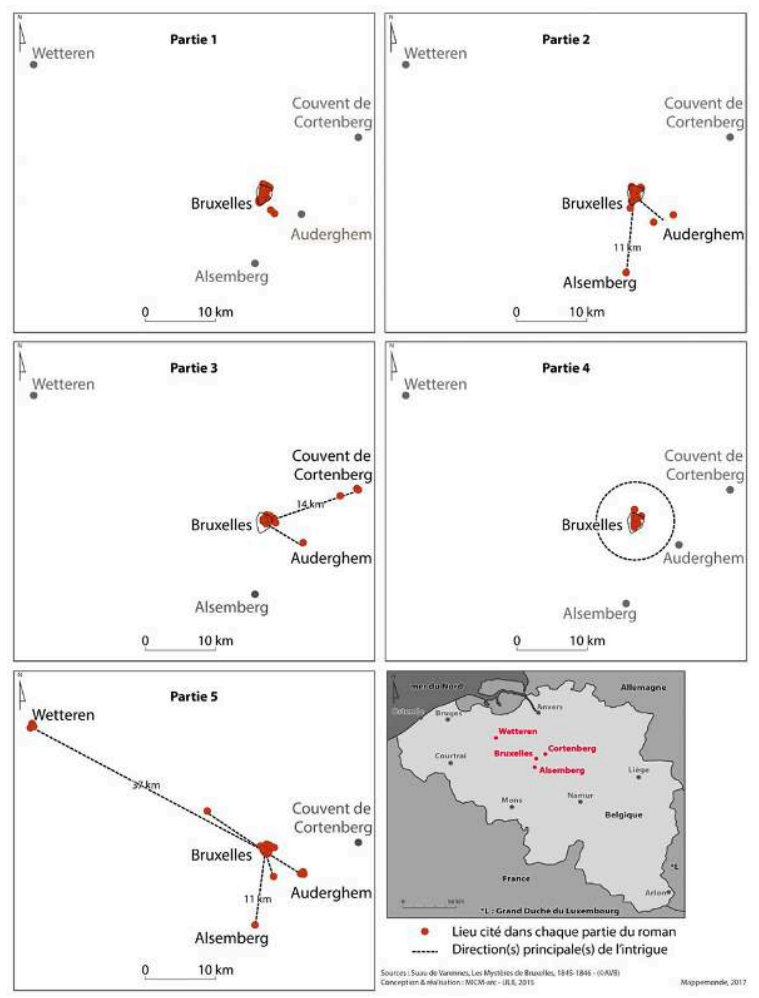

Proximité spatiale de groupes sociaux différenciés

22 Voyons d'abord l'ensemble des personnages. Une grille assez classique permet de les disposer selon un double axe horizontal (l'opposition bons vs mauvais) et vertical (selon leurs milieux sociaux, regroupés en trois "états" que seule la bonne volonté des puissants permet de dépasser). 
Figure 5. Schéma d'ensemble des personnages

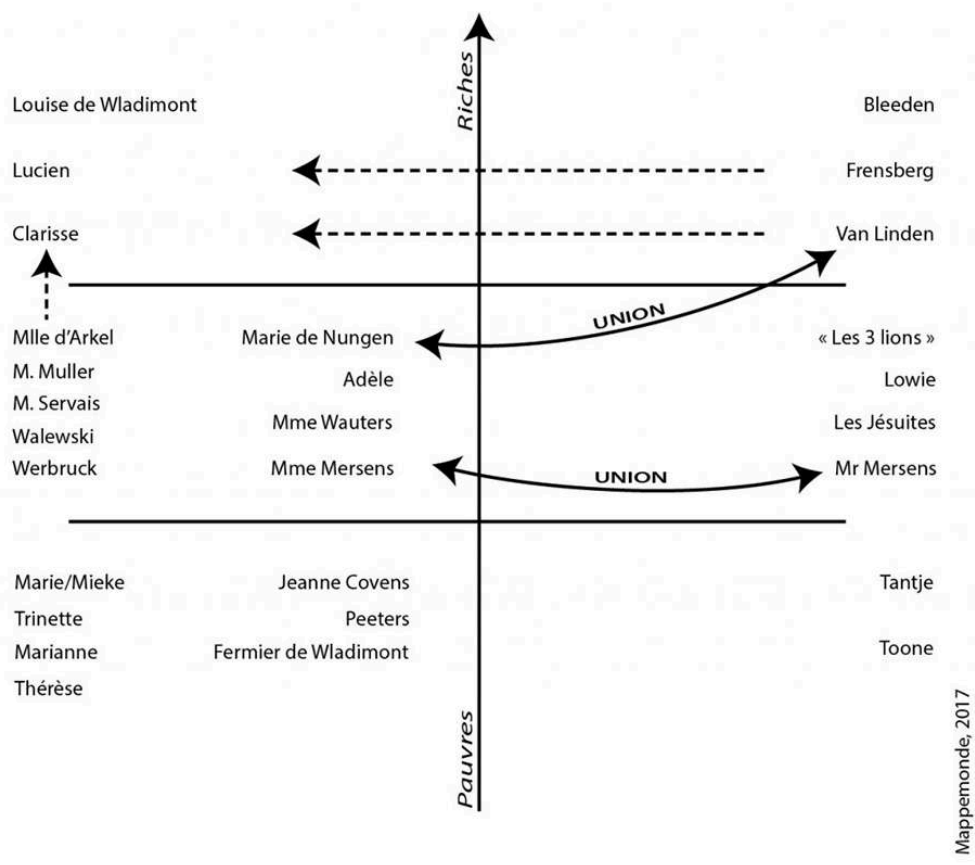

Les cartes permettent de reconstituer leurs parcours dans la ville. Il apparaît clairement qu'il s'agit d'un paramètre " difFérentialiste » pertinent.

Le roman décrit avec force détails les habitations particulières, tant dans leur localisation référentielle (quartier, rue) que dans leurs caractéristiques intérieures (décoration, mobilier, état général). L'esthétique du roman réaliste popularisée par Balzac fait en efFet communément du cadre de vie une métonymie du comportement et de la psychologie de ses habitants. Suau se borne ici à utiliser scrupuleusement un code littéraire bien connu. Mais ce choix demande à être interprété dans le contexte bruxellois de l'époque. La carte des lieux d'habitation découle directement de la base de données ; pour une lecture plus aisée, on a ajouté une représentation schématique du parcours de la rivière (la Senne), encore à ciel ouvert à l'époque, et le tracé des boulevards délimitant le Pentagone bruxellois : 
Figure 6. Carte des lieux de résidence des personnages

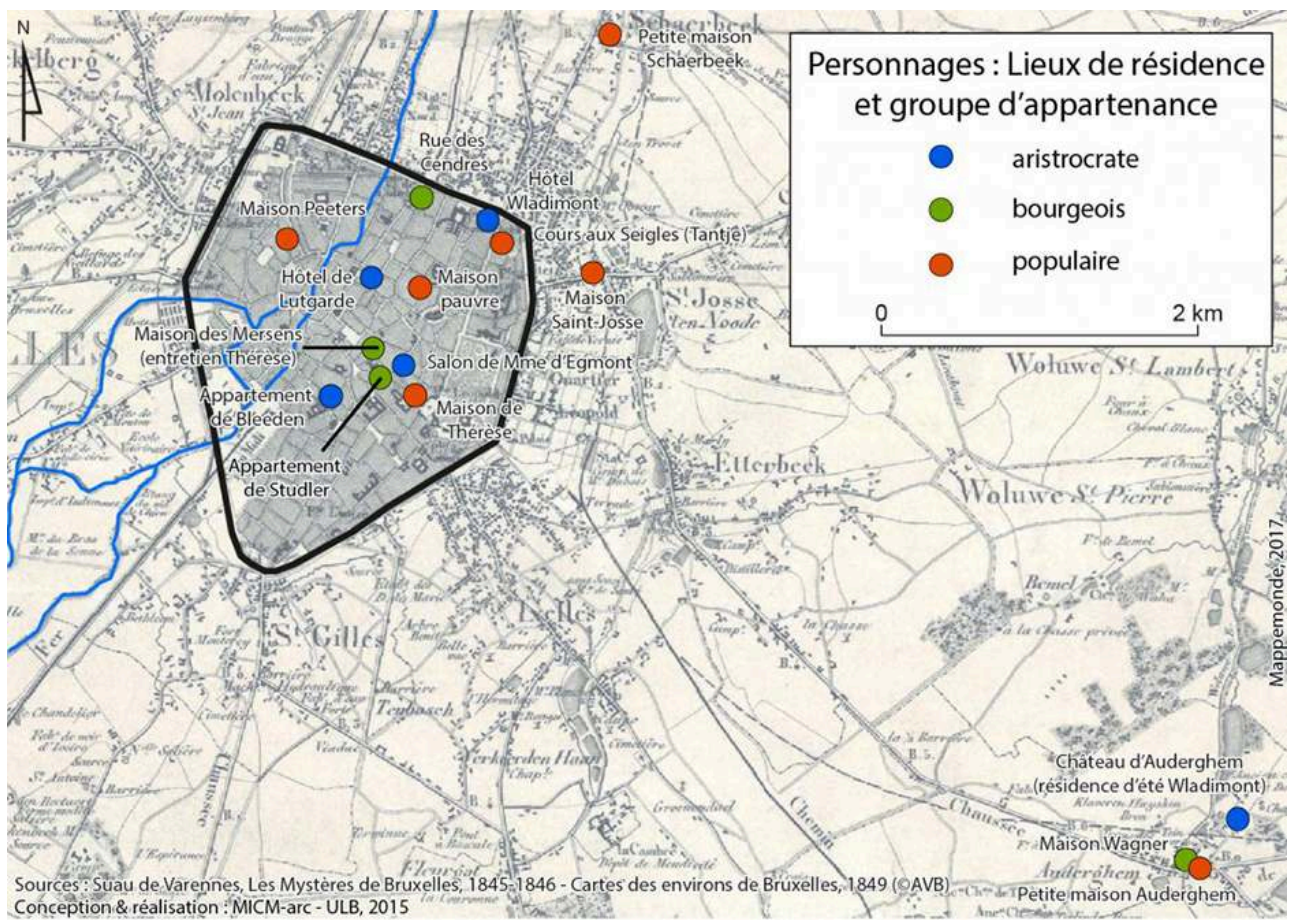

Une opposition nette apparaît entre l'habitat de la plupart des personnages et la maison du «bon ouvrier » Peeters, qui se situe près du port où il travaille, et dans un quartier qu'aucun autre personnage ne fréquente. Le «bon pauvre» étant une singularité dans le récit, la carte en matérialise ainsi l'exceptionnalité. Le roman de Suau montre ici tout ce qui le sépare du roman zolien, qui saura fusionner les lieux de l'action avec les lieux du travail ouvrier.

Une lecture anachronique tendrait à confondre l'ensemble des autres habitats dans un conglomérat urbain indifférencié, puisque les hôtels particuliers de l'aristocratie sont proches des taudis ouvriers. En réalité, Suau traduit ainsi une phase très précise de l'évolution d'une capitale au tout début de sa croissance. Dans les années 1840, la ville n'a en efFet pas encore commencé à s'étendre au-delà du Pentagone et la ville est en cours de ségrégation sociale, sans que les relations de proximité héritées de l'Ancien Régime aient totalement disparu. Comme on le verra, ce sont des micro-espaces urbains, à l'échelle d'une rue, d'un passage ou d'un boulevard qui sont alors signifiants, et les précisions spatiales du roman sont pleinement pertinentes si on les lit à cette échelle de détail et non à l'échelle d'une opposition entre quartiers.

Ce cadre historique permet de rappeler que la plupart des Mystères urbains ont été écrits, dans les innombrables pays où le genre a surgi, à un moment de recomposition de la ville ancienne. L'identification des classes dangereuses précède de peu les efForts faits pour les circonscrire spatialement, nous y reviendrons plus loin.

\section{Différenciation sociale par la mobilité}

Si le roman met en évidence les tensions surgissant dans une ville où les groupes sociaux se mêlent inextricablement dans un espace urbain encore peu ségrégué, il 
souligne néanmoins que les usages de la ville par les personnages, et en particulier leur mobilité, ne se font pas de la même manière.

La carte suivante suit le parcours d'un personnage particulier. La petite Mieke, qui est une sorte de Fleur-de-Marie (l'héroïne de Sue), représente le type de la pure jeune fille élevée dans le milieu social le plus paupérisé par une marâtre particulièrement cynique. Elle échappe au quartier de Notre-Dame-aux-Neiges (la Cour aux seigles), décrit comme le quartier le plus dangereux de Bruxelles (il sera détruit peu de temps après). Elle cherche de l'aide et se dirige spontanément vers le boulevard, plus aéré et cossu, puis vers le centre-ville, en suivant la pente naturelle de la vallée de la Senne. Son trajet est interrompu par l'autorité qui y a son siège et qui exerce là, dans le centre, un pouvoir qui s'impose plus faiblement à la périphérie. C'est aussi dans les quartiers centraux que la jeune fille espère trouver des personnes compatissantes, parce qu'ils sont fréquentés par une population plus variée socialement que celle des rues qu'elle emprunte d'abord. Son arrestation est la plus « spectaculaire " possible : elle a lieu sous les arcades du théâtre de la Monnaie, le haut lieu de la sociabilité bruxelloise, particulièrement protégé pour éviter que ne s'y manifestent trop explicitement les contrastes sociaux.

Figure 7. La fuite de Mieke

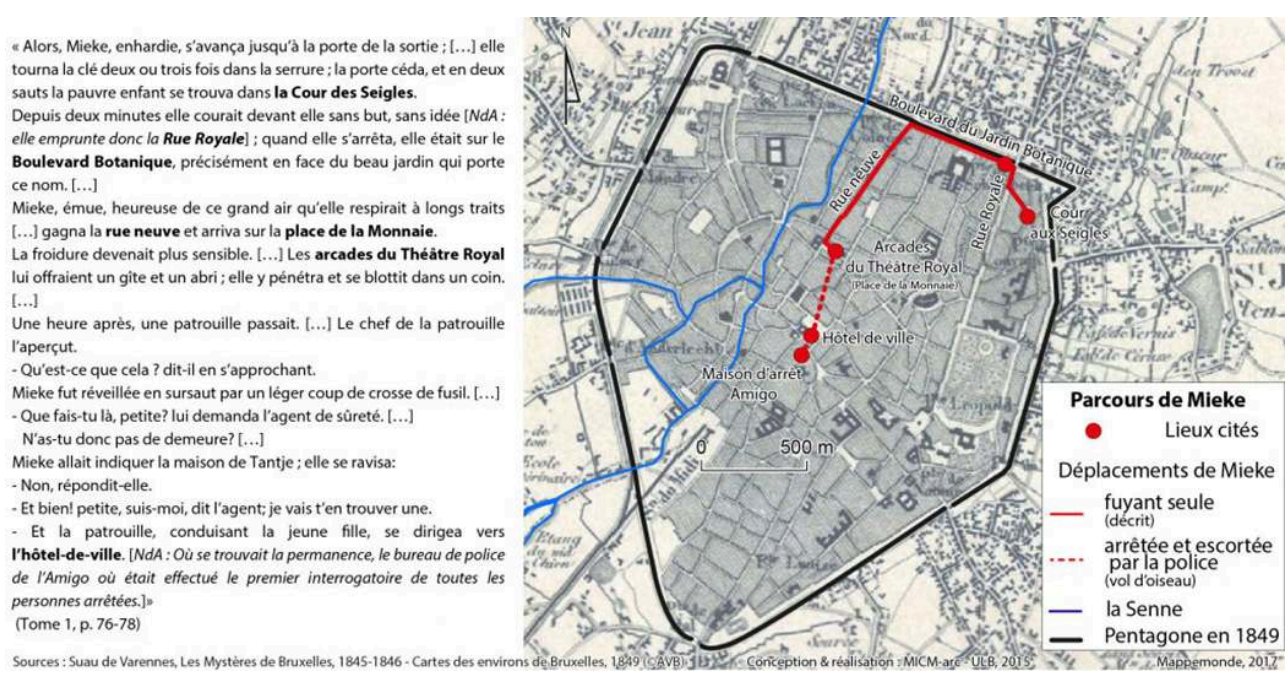

Les deux personnages principaux du roman, la duchesse de Wladimont et le chevalier de Bleeden sont également les personnages les plus mobiles. Ils ont les moyens d'occuper plusieurs lieux, leur habitation propre, mais aussi des cachettes diverses, et ils usent largement des moyens de transport les plus rapides qui existent alors. Comme le roman est pour une part une course-poursuite entre le Bien et le Mal, on ne s'étonnera pas de voir leurs trajectoires se suivre et se croiser à de nombreuses reprises. Toutefois le lecteur risque de ne pas prendre conscience que leurs déplacements si nombreux investissent des zones urbaines et périurbaines légèrement décalées. Si l'on prend en compte tous leurs trajets, il apparaît que le Mal se situe un peu plus au nord-ouest que le Bien. Bleeden franchit plus souvent la Senne que la duchesse, et il prépare ses mauvais coups en Flandre tandis que la duchesse séjourne au sud de Bruxelles, là où s'installera peu à peu la population aisée de la ville.

Le rapport à la mobilité est donc aussi un critère de difFérenciation ${ }^{10}$. Dans la ville du $\mathrm{XIX}^{\mathrm{e}}$ siècle se croisent toutes sortes de véhicules, qui sont liés à des fonctions précises 
(transport de marchandises ou de personnes, individuel ou collectif), à des statuts particuliers (propriété privée, ou location), à des connotations symboliques (un coupé n'est pas un landau), à des particularités constructives enfin (voiture couverte ou découverte, impliquant un cocher ou conduite par le propriétaire, etc. ${ }^{11}$. Les Mystères de Bruxelles donnent un inventaire très complet de ces types de transport. Il faut ajouter la marche à pied, qui reste le mode de déplacement de la majorité de la population, non par choix, mais par obligation - et on se souviendra que Mieke ne dispose évidemment d'aucun moyen de transport. Le roman précise bien qu'un aristocrate "se promène " tandis qu'un ouvrier «marche $»^{12}$. Le tableau suivant donne un aperçu général de la mobilité des personnages. Il permet de voir combien est inégalitaire la répartition des voitures attelées. Seule l'aristocratie en a l'usage, tandis que la bourgeoisie est réduite aux voitures de location (appelées « vigilantes»).

Tableau 2. Mobilité des personnages

\begin{tabular}{|c|c|c|c|c|c|c|c|c|c|c|c|c|}
\hline & Landau & Calecho & Berline & Coupe & Cabriolet & Tilbury & $\begin{array}{c}\text { Vigilantel } \\
\text { Fiacre }\end{array}$ & Carriole & charrette & Cheval & $\begin{array}{l}\text { Chemin } \\
\text { de fer }\end{array}$ & Marche \\
\hline Personnages & 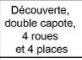 & $\begin{array}{c}\text { Decouverte, } \\
\text { capotte }\end{array}$ & $\begin{array}{l}\text { Fernés, } \\
4 \text { roues ot } \\
4 \text { placeses } \\
\end{array}$ & $\begin{array}{l}\text { Fermée. } \\
\text { 2rovos }\end{array}$ & 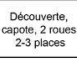 & $\begin{array}{c}\text { Decouverte, } \\
\text { leggrer, } 2 \text { rovous }\end{array}$ & $\begin{array}{c}\text { Voliue } \\
\text { pubifuo } \\
\end{array}$ & $\begin{array}{l}\text { Charerete } \\
\text { 2 roues }\end{array}$ & $\begin{array}{c}\text { Voture } \\
\text { compoganarde, } \\
\text { 2roues }\end{array}$ & & & \\
\hline $\begin{array}{l}\text { Duc de } \\
\text { Wladimont }\end{array}$ & $x$ & $x$ & $x$ & $x$ & & & & & & $x$ & & \\
\hline $\begin{array}{l}\text { Mme de } \\
\text { Wladimont }\end{array}$ & & $x$ & & $\mathrm{x}$ & & & & & & $x$ & & $\begin{array}{c}\mathrm{X}+ \\
\text { promenade }\end{array}$ \\
\hline $\begin{array}{l}\text { Marquise } \\
\text { d'Aspasie }\end{array}$ & & & $x$ & & & & & & & & & \\
\hline $\begin{array}{l}\text { Comte de } \\
\text { Frensberg }\end{array}$ & & & & $x$ & $x$ & & & & & $x$ & & \\
\hline $\begin{array}{l}\text { Comte } \\
\text { d'Epinoy }\end{array}$ & & & & $x$ & & & & & & $x$ & & \\
\hline 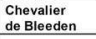 & & $x$ & & $x$ & & & & & & $x$ & & promenade \\
\hline de Fercourt & & & & & & & & (x) & & & & \\
\hline Van Linden & & & & & & & & (x) & & & & \\
\hline Lucien & & & & $x$ & & & & & & & & \\
\hline Alfred & & & & & & & & & & $x$ & & \\
\hline M. Mersens & & & & & & & & & & & & (x) \\
\hline Mme Mersens & & & & & & & $x$ & & & & & \\
\hline Lowie & & & & & & & & & & & $\mathrm{x}$ & $x$ \\
\hline Walewski & & & & & & & & & & & & $x$ \\
\hline $\begin{array}{l}\text { Les } \\
\text { "lionceaux" }\end{array}$ & & & & & & $x$ & & & & & & $x$ \\
\hline $\begin{array}{l}\begin{array}{l}\text { Parents } \\
\text { d'Adèle }\end{array} \\
\end{array}$ & & & & & & & & & & & & $x$ \\
\hline $\begin{array}{l}\text { Sociétaires } \\
\text { du } B A C\end{array}$ & & & & & & $x$ & & & & & & \\
\hline $\begin{array}{l}\text { Deux } \\
\text { Français }\end{array}$ & & & & & & & $\mathrm{x}$ & & & & & $\mathrm{x}$ \\
\hline Studler & & & $(x)$ & & & & $x$ & & & & $x$ & \\
\hline Theyssens & & & & & & & $x$ & & & & & \\
\hline Peeters & & & & (x) & & & $x$ & & & & & \begin{tabular}{|c|}
$x+$ \\
promenade
\end{tabular} \\
\hline $\begin{array}{l}\text { Toone } \\
\text { et Henri }\end{array}$ & & & & & & & $x$ & & & & & $\mathrm{x}$ \\
\hline Marie/Mieke & & & & & & & $(x)$ & & & & & $x$ \\
\hline Paysan & & & & & & & & & $x$ & & & \\
\hline
\end{tabular}

\section{Comment l'espace enrichit-il le sens du récit ?}

Le lecteur attentif à suivre les lieux de rencontres entre les personnages remarque sans peine ceux que Suau privilégie. Dans l'ordre du récit, il s'agit en particulier de l'hôtel Cluysenaar, d'un marchand de tabac et d'une rue. Nous les traiterons en ordre inverse, pour respecter leur importance relative. Il est permis de parler à leur sujet de chronotopes thématiques, au sens de Henri Mitterand, lorsqu'on a en vue leur fonction littéraire. Mais lorsque nous reportons ces chronotopes sur la carte, nous en faisons pleinement des objets d'étude historique, et enrichissons de la sorte le sens du texte. 


\section{Une rue huppée}

Une rue quasiment disparue de nos jours prend une importance particulière dans notre base de données : la rue Montagne-de-la-Cour. On retrouve cette rue à divers moments $\mathrm{du}$ récit, avec les mêmes connotations de luxe et de lieu de rencontres pour la bonne société. Les voleurs se félicitent d'y avoir pu piller une boutique (I, p. 234) ; les jeunes aristocrates s'y promènent - « en se dandinant nonchalamment » précise le texte (I, p. 241 ) - et c'est là que l'un d'eux croit rencontrer une bonne fortune en croisant une voiture attelée qui grimpe péniblement la pente. Enfin, le lieu représente le sommet de l'ascension sociale de $\mathrm{M}^{\text {me }}$ Wouters et de ses filles, les tenancières du bureau de tabac devenues, grâce à la Duchesse de Wladimont, les propriétaires d'un magasin de lingerie fine.

Il est symptomatique qu'un roman voué à explorer les contrastes urbains privilégie une artère aussi luxueuse : il rend ainsi compte d'un imaginaire social partagé par toutes les couches de la société, mais évidemment très inégalement réparti entre elles.

\section{Un magasin à la mode}

Un second lieu stratégique du récit est le magasin de tabac que tient Madame Wouters, rue de la Madeleine. Étape précédente de son parcours social, elle se situe précisément dans la rue qui se trouve immédiatement en contrebas de la Montagne-de-la-Cour. Elle est à l'époque, selon Suau, une rue passante méritant d'être décrite.

Ce n'est pas un hasard si une des premières représentations des rues de Bruxelles sous la forme d'un panorama prend précisément pour sujet la rue de la Madeleine.

Figure 8. La rue de la Madeleine

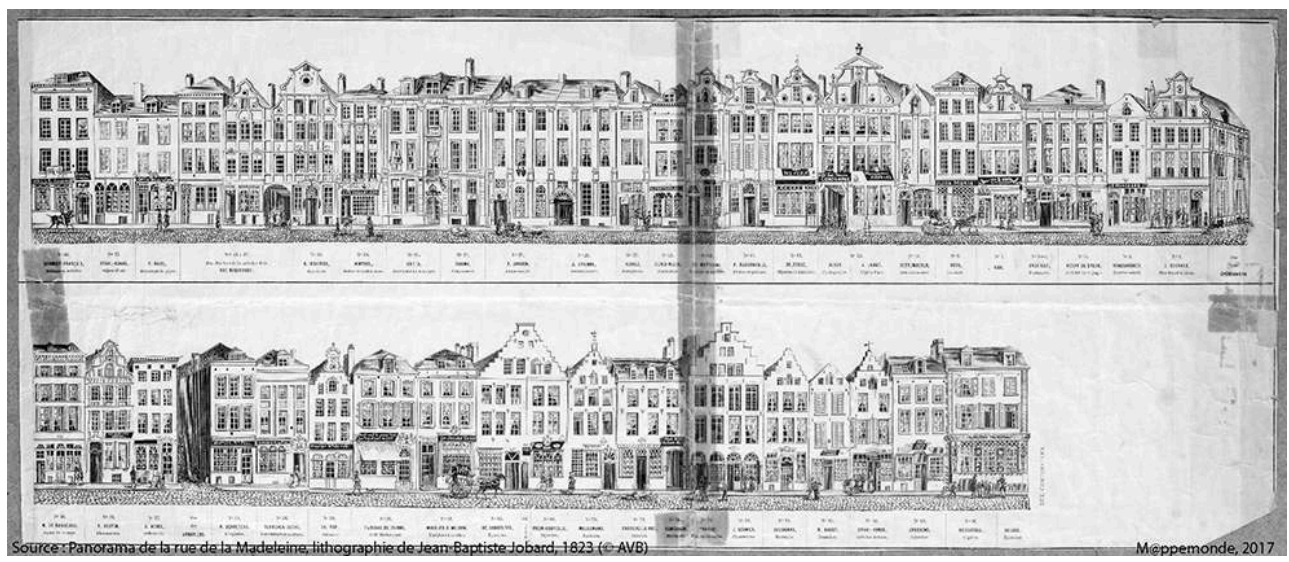

Le magasin de tabac a ceci de particulier qu'il est fréquenté par des hommes et tenu par des femmes - après avoir été la victime des "méchants ", $\mathrm{M}^{\mathrm{me}}$ Wouters changera de clientèle autant que de statut social. Le roman précise qu'il ne s'agit pas seulement d'un lieu d'achat, mais aussi d'une sorte de salon où l'on s'arrête longuement pour parler entre gens du même monde, en l'occurrence les jeunes « lions » à la mode (I, p. 106).

Le contexte explique les « airs de vanité » qu'ils arborent. Le cigare, en tant que tabac à fumer importé des colonies, est en efFet l'objet d'une mode récente. Il s'oppose au tabac à priser de l'Ancien Régime, et implique une mainmise olfactive masculine sur l'espace ambiant, voire la création de rituels et d'espaces dédiés dans les intérieurs (le 
fumoir). Il devient dès lors rapidement un signe de distinction sociale, renforcé par l'invention contemporaine des bagues qui signalent aux spectateurs la marque (et donc le prix) de l'objet. Le chronotope régit dès lors les rencontres romanesques : «Un jour que le hasard avait conduit le chevalier dans le magasin de madame Wouters pour y faire quelques emplettes de cigares [...]» (I, p. 139); un hasard bien situé, au lieu précis où se rencontrent la ville basse et la ville haute $!^{13}$

\section{Un mauvais quartier à assainir}

Nous avons déjà signalé l'importance symbolique de l'hôtel Cluysenaar, situé 75 rue Royale neuve, dont la description ouvre le roman. Il offre l'intérêt de décrire un bâtiment réel, dans lequel il est probable que l'auteur ait lui-même logé.

Figure 9. L'hôtel Cluysenaar, rue Royale

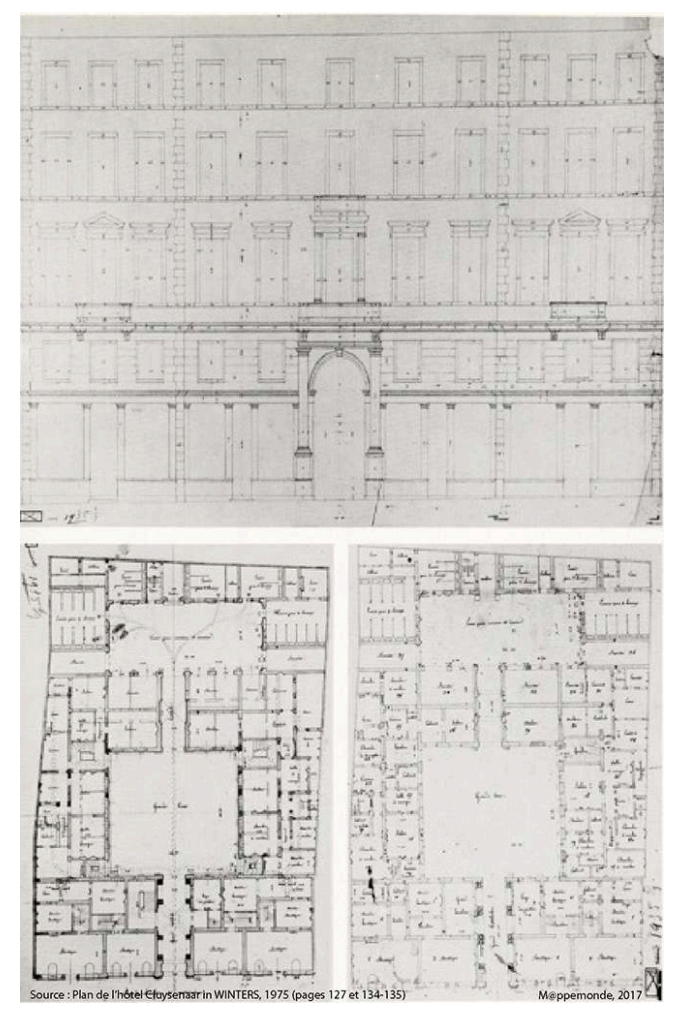

La situation de ce bâtiment est caractéristique des tensions sociales bruxelloises: construit le long d'une route nouvelle destinée aux classes dominantes, et qui annonce les grands travaux d'assainissement de la capitale, il jouxte deux des quartiers pauvres (et donc dangereux) : le quartier du Marais, vers le bas de la ville, et le quartier de Notre-Dame-aux-Neiges, à l'est de la rue Royale.

41 L'hôtel Cluysenaar devient ainsi le point de départ d'une plongée dans une capitale en pleine évolution démographique et urbanistique, qui conserve ses mauvais quartiers, ses criminels endurcis, ses milieux populaires et ses lieux de misère, en regard de beaux et nouveaux aménagements urbains. Son nom même fait le lien entre le constat de la misère sociale et les propositions urbanistiques censées embellir la ville et en rénover l'habitat. 
Jean-Pierre Cluysenaar (1811-1880) était à la fois un architecte (d'obédience néoclassique et éclectique) et un spéculateur foncier très entreprenant. Outre l'hôtel qui porte son nom, il est particulièrement actif en plusieurs lieux du roman de Suau : rue de la Madeleine, où il bâtit un marché en 1847, rue Royale, où il organise le panorama de la colonne du Congrès (1847).

Suau vivait déjà à Bruxelles lorsque, le 7 septembre 1843, le Ministère de l'Intérieur installa une commission chargée de faire un rapport sur «la condition des classes ouvrières et sur le travail des enfants ». La personnalité la plus active de cette enquête était Édouard Ducpétiaux (1804-1868). Cet ancien journaliste fut nommé en 1830 inspecteur général des prisons et des instituts de bienfaisance. Sans attendre la conclusion de l'enquête, qui parut en 1848, il publia de nombreux travaux, dont De la condition physique et morale des jeunes ouvriers et des moyens de l'améliorer en 1843 et $\mathrm{Du}$ paupérisme en Belgique. Causes et remèdes en 1844. Suau puisa une grande part de son inspiration dans ces ouvrages, ainsi que dans les travaux des statisticiens, une science en développement dont le Belge Adolphe Quetelet était un des principaux chefs de file. Ce dernier publia en 1842 une carte de l'indigence à Bruxelles fondée sur les données du recensement de la population. C'était une des premières applications de la cartographie statistique.

Nous pouvons dès lors représenter sur un même support les lieux cités par le roman, les constructions de Cluysenaar et les données statistiques révélées par Quetelet.

Figure 10. Carte de l'indigence à Bruxelles

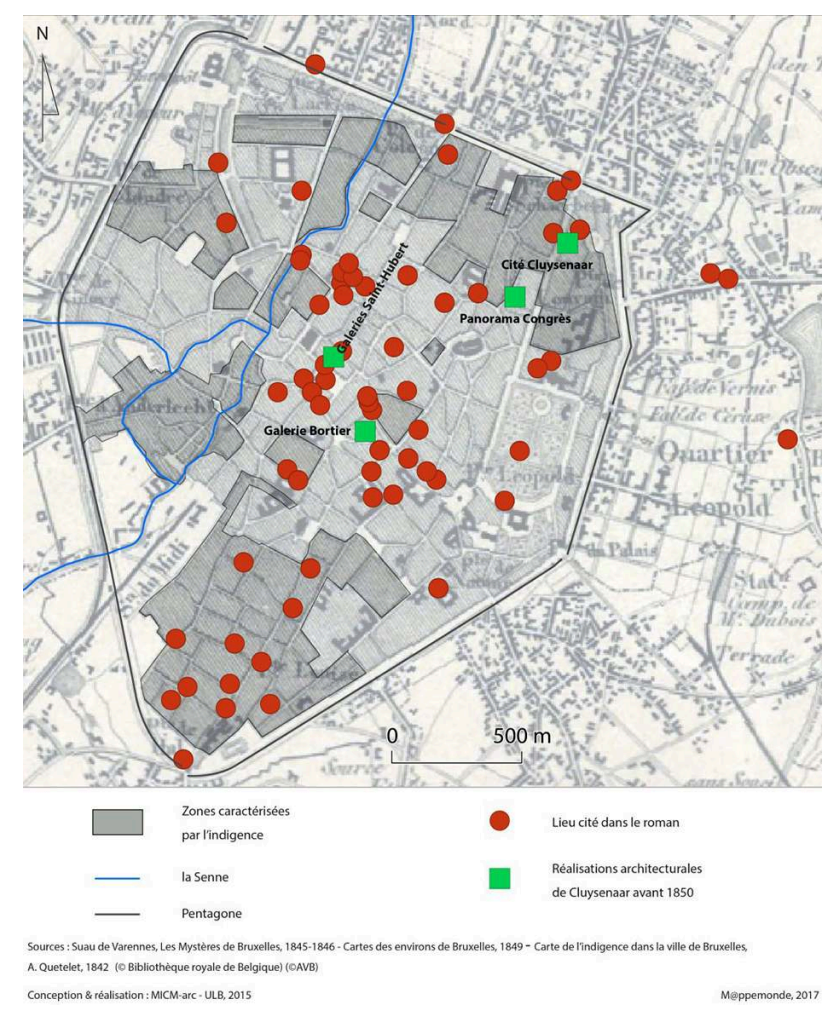

Le roman philanthropique des Mystères ne se borne pas à accompagner un mouvement de pensée, comme on l'a souvent dit, il s'ancre dans l'espace urbain de la manière la plus concrète, et la carte matérialise sous nos yeux la relative convergence de l'action simultanée des discours sociaux de l'enquête, de l'architecture et de la fiction. 


\section{Conclusion} sans recourir nécessairement à l'analyse quantitative - des structures spatiales récurrentes dans le récit. Elles le permettent d'autant plus facilement qu'elles constituent un terrain propice au dialogue interdisciplinaire. En tant que représentations univoques des espaces romanesques, pouvant être examinées de concert, elles donnent en efFet la possibilité de mettre en commun les observations et interprétations propres à chaque discipline du traitement difFérencié que le texte réserve au territoire auquel il fait référence. Dans le cas des Mystères de Bruxelles, la représentation cartographique a notamment permis à l'équipe de mettre à jour que le récit était construit sur une opposition entre la ville centre et sa périphérie ou qu'il mettait en exergue la proximité spatiale des groupes sociaux dans un espace urbain encore faiblement ségrégué. S’il est évident qu'un bon lecteur peut souligner ces aspects sans recourir à la cartographie, il est non moins évident que celle-ci leur donne une dimension systématique qui les met en évidence. En ce sens, la mise en cartes a été le révélateur des chronotopes structurels d'espèce et de sous-espèce et des chronotopes thématiques des Mystères de Bruxelles

Au terme de cet exercice, il apparaît que le chronotope se révèle une grille d'analyse particulièrement pertinente des « espaces-temps » d'un roman, à la condition d'en bien différencier les niveaux d'application. Le tableau qui suit synthétise nos propositions en ce qui concerne les Mystères de Bruxelles. Il sépare les chronotopes que nous avons retenus en deux catégories, selon que l'on prend en considération les catégories génériques du littéraire ou les lieux/moments privilégiés par le modèle romanesque particulier auquel nous avons afFaire. Nous reprenons à Henri Mitterand la distinction en genre et espèce et introduisons la catégorie de sous-espèce pour le roman des Mystères de. Dans ce cadre, nous rappelons les chronotopes thématiques qui nous ont paru pertinents. 
Tableau 3. Tableau de synthèse des chronotopes du roman

\begin{tabular}{|c|c|c|c|}
\hline $\begin{array}{l}\text { Groupe de } \\
\text { chronotopes }\end{array}$ & $\begin{array}{l}\text { Catégorie de } \\
\text { chronotope }\end{array}$ & $\begin{array}{l}\text { Les mystères } \\
\text { de Bruxelles }\end{array}$ & Commentaires \\
\hline & Genre & Roman & $\begin{array}{l}\text { Le roman est un genre permettant l'introduction } \\
\text { de temps et de lieux concrets: il se développe } \\
\text { particulièrement depuis le début du XIX siècle. }\end{array}$ \\
\hline \multirow[t]{3}{*}{ Structurels } & Espèce & $\begin{array}{l}\text { Roman } \\
\text { urbain }\end{array}$ & $\begin{array}{l}\text { Il se déploie dans l'espace de la grande ville } \\
\text { contemporain à l'auteur. }\end{array}$ \\
\hline & Sous-espèce & Mystère de & $\begin{array}{l}\text { Le genre de Mystères de est initié par E. Sue en } \\
1842-1843 \text {, qui y décrit Paris. Il met } \\
\text { particulièrement en évidence des tensions } \\
\text { sociales et la dynamique urbaine du XIX }{ }^{\mathrm{e}} \text { siècle. }\end{array}$ \\
\hline & Rue huppée & $\begin{array}{l}\text { Rue } \\
\text { Montagne de } \\
\text { la Cour }\end{array}$ & $\begin{array}{l}\text { Lieu/moment de rencontre et de loisir ; symbole } \\
\text { d'une ascension sociale. }\end{array}$ \\
\hline \multirow[t]{2}{*}{ Thématiques } & $\begin{array}{l}\text { Commerce à la } \\
\text { mode }\end{array}$ & $\begin{array}{l}\text { Magasin de } \\
\text { tabac }\end{array}$ & $\begin{array}{l}\text { Lieu/moment typique de la masculinité } \\
\text { mondaine }\end{array}$ \\
\hline & $\begin{array}{l}\text { Mauvais } \\
\text { quartier } \\
\text { assainir }\end{array}$ & $\begin{array}{l}\text { Notre-Dame- } \\
\text { aux-Neiges }\end{array}$ & Lieu/moment de la dynamique spéculative. \\
\hline
\end{tabular}

Source : Suau de Varennes, Les Mystères de Bruxelles, 1845-1846. Conception \& réalisation : MICMarc-ULB, 2015. M@ppemonde, 2017 interprétation historique (on en a d'ailleurs fait le reproche à Bakhtine). Celle-ci va bien au-delà du "contexte», parce qu'elle montre combien tous les événements fictionnels gagnent à être perçus de manière référentielle. C'est ici que l'interprétation pluridisciplinaire est surtout utile. Qu'une des modalités de la lutte des classes soit une lutte pour la mobilité amène à considérer d'un autre œil les parcours des personnages : apport de l'historien. Que la recomposition urbanistique, fonctionnelle et sociale de Bruxelles au XIX ${ }^{e}$ siècle contribua à faire émerger de nouvelles différenciations spatiales conduit à donner du sens à l'inscription contrastée du matériau romanesque dans le territoire : apport du géographe. Inversement, rappeler que le roman décrit une action et non seulement des lieux implique de se donner les moyens de décrire le sens du récit : apport du « littéraire».

51 En définitive, on le voit, l'usage que nous faisons de la carte est bien éloigné du simple repérage des lieux désignés par un texte. Sa dimension heuristique l'emporte sur la dimension représentative. Elle devient à ce titre un vecteur efficace de l'herméneutique textuelle. 


\section{BIBLIOGRAPHIE}

ARON P. (1997). « Leclercq, Pierre-Joseph, Émile ». In Nouvelle Biographie nationale, Bruxelles, Académie royale des Sciences, des Lettres et des Beaux-Arts de Belgique, t. 4, p. 242-243.

ARON P. (2015). « Les mystères des Mystères de Bruxelles ». Médias 19, « Mysterymania », KALIFA D., THERENTY M.-È. (dir.), Les Mystères urbains au XIXe siècle : Circulations, transferts, appropriations, mis à jour le 19/02/2015. http://www.medias19.org/index.php?id=17993

BAKHTINE M. (1978). « Formes du temps et du chronotope dans le roman ». In Esthétique et théorie du roman (1937), trad. française, Paris : Gallimard.

BÉGUIN M., PUMAIN D. (1994). La représentation des données géographiques. Paris : Armand Colin, coll. «Cursus».

BEMONG N., BORGHART P., De DOBbeleER M., DEMOEN K., DE TEMMERMAN K., KEUNEN B. (éd.) (2010). Bakhtin's Theory of the Literary Chronotope: Reflections, Applications, Perspectives. Gent: Academia Press, 250 p. ISBN 9789038215631

BILLEN C. (2015). « De l'Avocat Richard aux Scrupules de Bernus, le désenchantement bruxellois d'Émile Leclercq (1817-1907)». Textyles, n 47, p. 69-81. https://journals.openedition.org/ textyles $/ 2630$

BRUNET R. (1987). La carte mode d'emploi. Paris/Montpellier : Fayard/RECLUS.

Collot M. (2014). Pour une géographie littéraire. Paris : Éditions Corti, coll. « Les Essais », 270 p. ISBN 9782714311290

FERRIER A. (1841). Guide pittoresque du voyageur en Belgique. Bruxelles : Hauman \& $C^{\mathrm{ie}}, 292 \mathrm{p}$.

FLONNEAU M., GUIGUENO V. (éd.) (2009). De l'histoire des transports à l'histoire de la mobilité ? Rennes : Presses Universitaires de Rennes, 334 p. ISBN 9782753509085

GROUPE $\mu$ (1990). Rhétorique de la poésie. Lecture tabulaire, lecture linéaire. Paris : Seuil, coll. « Points », $384 \mathrm{p}$.

JAUMAIN J., Loir C. (éd.) (2003). « Actes de la journée d'étude : les guides de voyage : une source pour l'histoire de Belgique ». Archives et Bibliothèques de Belgique, t. LXXIV, n 1-4, p. 11-204. https://difusion.ulb.ac.be/vufind/Record/ULB-DIPOT:oai:dipot.ulb.ac.be:2013/68380/Holdings HAMON Ph. (1972). « Pour un statut sémiologique du personnage ». Littérature, vol. 6, n 6, p. 86-110.

HORVATH C. (2008). Le Roman urbain contemporain en France. Paris : Presses de la Sorbonne Nouvelle. ISBN 9782878543773

LOIR C., TURCOT L. (éd.) (2011). La promenade au tournant des XVIII et XIX ${ }^{e}$ siècles. Belgique - France Angleterre. Bruxelles : Éditions de l'Université de Bruxelles, coll. «Études sur le $18^{\mathrm{e}}$ siècle », 256 p. ISBN 9782800415123

MASSERON C., PETITJEAN B. (1979). « Pour une définition du personnage : l'exemple de Germinal ». Pratiques, $\mathrm{n}^{\circ}$ 22-23, mars, p. 69-96.

MEINER C. (2008). Le carrosse littéraire et l'invention du hasard. Paris : PUF, coll. « Les littéraires ", 250 p. ISBN 9782130564188 
MitTERAND H. (1990). «Chronotopies romanesques : Germinal ». Poétique, n 81, p. 89-104.

MONTANDON A. (1998). « Pour une sociopoétique du chronotope : la scène de bal chez Théophile Gautier ». Littérature, vol. 112, p. 14-25.

ROSSETTO T. (2013). « Theorizing maps with literature ». Progress in Human Geography, vol. 38, $n^{\circ} 4$. https://journals.sagepub.com/doi/full/10.1177/0309132513510587

SAMINADAYAR-PERRIN (éd.) (2013). Les Mystères urbains au XIXe siècle : le roman de l'histoire sociale. Autour de Vallès, nº43.

SUAU DE VARENNES E. (1845-1846). Les mystères de Bruxelles. Bruxelles : Société typographique belge, A. Wahlen, $5 \mathrm{t}$.

TURCOT L. (2007). Le promeneur à Paris au XVIII siècle. Paris: Gallimard, 440 p. ISBN 9782070783663

WINTERS J. (1981). « Maatschappij en maatschappijbeeld : Jean-Pierre Cluysenaar ». Openbaar

Kunstbezit Vlaanderen, XIII, p. 123-142.

ZANIN C. (2006). « Cartographie thématique ». Hypergeo. http://www.hypergeo.eu/spip.php? article377

\section{NOTES}

1. On pense ici au concept de « lecture tabulaire » proposé par le Groupe $\mu$ (1990).

2. Sur les diverses définitions du chronotope, voir Mitterand (1990); Montandon (1998) et Bemong (2010).

3. Voir Rossetto (2013). On peut lire une bonne synthèse des tendances actuelles dans Collot (2014).

4. En l'occurrence le logiciel libre QGIS.

5. Sur les avantages qu'offrent la cartographie thématique pour l'analyse des répartitions spatiales voir Brunet (1987); Béguin et Pumain (1994).

6. Saminadayar-Perrin (2013).

7. Les adresses ont été localisées grâce aux cartes et index de rues conservées aux Archives de la Ville de Bruxelles, puis cartographiées via QGIS.

8. Des ouvrages spécialisés destinés à faire découvrir Bruxelles aux visiteurs sont publiés dès la fin du $18^{\mathrm{e}}$ siècle, bien avant le premier Baedeker consacré à la Belgique et à la Hollande (1869). Ces ouvrages dressent le catalogue des principaux monuments qu'il convient de visiter, l'inventaire des collection d'art, les promenades et les meilleures ressources de la ville (Jaumain et Loir, 2003).

9. Voir les études classiques de Hamon (1972) et de Masseron C., Petitjean B. (1979).

10. Sur l'histoire de la mobilité, voir Flonneau, Guigueno (2009).

11. Voir les analyses suggestives de Meiner (2008).

12. Sur la promenade, voir Turcot (2007) et Loir, Turcot (2011).

13. Un récit plus tardif du romancier réaliste Émile Leclercq tourne, lui, entièrement autour d'une boutique de la rue de la Madeleine. Voir Émile Leclercq, Histoire de deux armurières (1864) et sur cet auteur, Aron (1997) et Billen (2015). 


\section{RÉSUMÉS}

Une des premières ambitions du roman urbain qui s'impose au milieu du $19^{\mathrm{e}}$ siècle est de donner à lire la ville dans sa structure et sa composition sociale. Ce genre romanesque a été étudié sur le plan thématique, mais également à la lumière du concept de chronotope bakhtinien. L'étude classique de Franco Moretti (2000) a toutefois initié un «tournant géographique » à son propos, qu'il semble utile de prolonger en confrontant ses apports à l'analyse chronotopique. En premier lieu, il importe de sortir d'une description globale du roman pour s'approcher au plus près de la dynamique même d'un récit singulier (rythme de l'histoire, parcours et rencontres des personnages dans l'espace). On essayera aussi d'indiquer ce que la cartographie apporte à une lecture attentive du roman. En quoi et comment la production et la visualisation par les cartes génèrent-elles un savoir neuf, impensé et impensable par l'analyse chronotopique ? L'article suit ce double programme en prenant pour objet Les Mystères de Bruxelles (1845-1846) de Suau de Varennes, roman urbain exhumé de l'oubli qui relève des multiples imitations des Mystères de Paris. On verra ainsi que la cartographie permet de réévaluer une œuvre méconnue, tout en indiquant, sur le plan méthodologique, quelques pistes fécondes de la rencontre entre l'histoire sociale, l'histoire littéraire et la géographie urbaine.

The urban novels that developed during the second half of the $19^{\text {th }}$ century aimed to present the structure and social composition of the city. Such novels have been analysed mainly through thematic analysis, but also with the Bakhtinian chronotope. Nevertheless, Franco Moretti's (2000) now classic study initiated a 'geographical turn' that calls for a more systematic analysis of what it contributes to the chronotopic approach. Firstly, beyond the overall description of the novel, it is interesting to focus on the dynamics of specific stories described within the novel (the story's pace, the routes and encounters of the various characters). Secondly, we will also examine cartography's contribution to the careful reading of a novel. How do mapping and visualisation through maps allow for a new knowledge, which was inconceivable and inconceived using chronotope analysis? This contribution considers both these dimensions in Les Mystères de Bruxelles (1845-1846) by Suau de Varennes, an urban novel rediscovered recently that is a variation of the well-known Mystères de Paris. Cartography allows a reassessment of an unknown literary work. It also opens up fertile prospects for collaborative research between social history, literature and urban geography.

Uno de los objetivos la novela urbana de mediados del siglo XIX era mostrar la estructura y composición social de las ciudades. Este género novelesco ha sido ampliamente estudiando, y se puede abordar bajo los principios del concepto de cronotopo expuesto por Bajtín. Si los estudios clásicos de Franco Moretti (2000) supusieron un giro geográfico, éstos se pueden confrontar con el análisis cronotópico. Por una parte, porque hay que salir de una descripción global de la novela para aproximarse de forma más cercana a la dinámica del relato (ritmo de la historia, recorridos y encuentro de los personajes en el espacio). Por otra, para valorar en qué medida la cartografía refuerza y mejora la lectura de la novela ; y en qué y cómo la elaboración y la visualización de los mapas generan un conocimiento nuevo no previsto por el análisis cronotópico. El artículo considera estas dos propuestas analizando los Misterios de Bruselas (1845-1846) de Suau de Varennes, novela urbana recuperada del olvido, y una las múltiples imitaciones de los Misterios de París. La cartografía permite estudiar desde otras metodologías una obra desconocida, ofreciendo nuevas líneas de investigación de encuentro entre la historia social, la historia literaria y la geografía urbana 
INDEX

Thèmes : La mise en carte des récits

Palabras claves : novela urbana, cronotopo, cartografías narrativas, Misterios, Bruselas

Keywords : urban novel, chronotype, narrative cartography, Mystères, Brussels

Mots-clés : Bruxelles, cartographie narrative, chronotope, Mystères, roman urbain

\section{AUTEURS}

\section{PAUL ARON}

Université libre de Bruxelles

\section{LAURENCE BROGNIEZ}

Université libre de Bruxelles

\section{TATIANA DEBROUX}

Université libre de Bruxelles

JEAN-MICHEL DECROLY

Université libre de Bruxelles

\section{CHRISTOPHE LOIR}

Université libre de Bruxelles 